\title{
MOLECULAR DYNAMICS STUDY OF SHORT AND MEDIUM RANGE ORDER IN MODIFIED BGO GLASSES
}

\author{
ADAM WALENTYNOWICZ1, AGNIESZKA WITKOWSKA ${ }^{1,2}$, MICHAL BIALOSKÓRSKI ${ }^{1,2}$, \\ JAROSŁAW RYBICKI ${ }^{1,2 *}$, SANDRO FELIZIANI ${ }^{3}$, SANDRO FRIGIO ${ }^{3}$ AND GIANCARLO COSIMI ${ }^{3}$ \\ ${ }^{1}$ Department of Solid State Physics, Faculty of Technical Physics and Applied Mathematics \\ Gdansk University of Technology, Narutowicza 11/12, 80-952 Gdańsk, Poland \\ ${ }^{2}$ TASK Computer Centre, Narutowicza 11/12, 80-952 Gdańsk, Poland \\ ${ }^{3}$ Istituto di Matematica e Informatica, Universita di Camerino, Camerino (MC), Italia \\ (Rec. 19 May 2004)
}

\begin{abstract}
In the paper we present our recent Molecular Dynamics (MD) simulations of the structure of bismuth-germanate (BGO) glasses of $x\left[p \mathrm{Bi}_{2}(1-p) \mathrm{Bi}_{2} \mathrm{O}_{3}\right](1-x) \mathrm{GeO}_{2}$ composition, where $x$ denotes the content of the bismuth oxide in unmodified glasses, and $p$ - the fraction of neutral bismuth that can appear in the surface modification process (e.g. annealing in hydrogen atmosphere). We consider glasses of compositions $x=0.1,0.2,0.3$ and reduction degrees $p=0,0.25,0.5,0.75,1$. The simulation results are analysed in detail and compared with the structural data provided by other authors.
\end{abstract}

\section{INTRODUCTION}

Although silicate and germanate glasses containing oxides of heavy metals such as $\mathrm{PbO}$, $\mathrm{Bi}_{2} \mathrm{O}_{3}, \mathrm{Sb}_{2} \mathrm{O}_{3}$, have been studied for many years due to their interesting optical properties, they are still promissing candidates for new materials to be applied in future non-linear optical and magneto-optical devices [1, 2]. These glasses, suitably modified (e.g. by annealing in hydrogen atmosphere), contain confined metallic nano-granules and exhibit very high secondaryemission coefficient and surface conductivity, thus finding applications in the production of electron channel multipliers [3]. The present paper is dedicated to the structure of bismuthgermanate glasses.

So far, most of the work done on $\mathrm{Bi}_{2} \mathrm{O}_{3}-\mathrm{GeO}_{2}$ (BGO) compounds has concerned $\mathrm{Bi}_{4} \mathrm{Ge}_{3} \mathrm{O}_{12}$ and $\mathrm{Bi}_{12} \mathrm{GeO}_{20}$ crystals, applied in scintillators and optoelectronical devices. The structure and properties of these materials are extensively described in [4-6]. Bismuth-germanate glasses have been investigated to a lesser extend [6-11].

It should be noted that bismuth-germanate glasses are highly susceptibile to structural modifications in the near-surface layer. Such structural modifications can be produced e.g. by high-temperature annealing in hydrogen atmosphere or by proton bombardment. As a result, bismuth ions become neutral atoms of high mobility, and form metallic granules in a germania

\footnotetext{
* Corresponding author: e-mail: ryba@pg.gda.pl
} 
matrix. The appearance of a granular structure in the surface layer is responsible for a significant increase in surface electrical conductivity [12-14], a very high secondary emission coefficient [13], and changes in X-ray absorption spectra (XAFS) [11].

In the present paper we present the results of our recent Molecular Dynamics (MD) simulations of the structure of $x\left[p \mathrm{Bi}_{2}(1-p) \mathrm{Bi}_{2} \mathrm{O}_{3}\right](1-x) \mathrm{GeO}_{2}$ glasses, where $x$ denotes the content of the bismuth oxide in unmodified glasses, and $p$ - the modification (reduction) degree, i.e. the fraction of neutral bismuth that appears in the annealing process. We consider glasses of compositions $x=0.1,0.2,0.3$ and modification degrees $p=0,0.25,0.5,0.75,1.0$. Such values of $x$ cover the glass-formation range of the considered glasses.

The paper is organised as follows. In Section 2 we describe in general the performed simulations and the methods of structural analysis we have used. The short and medium-range orders of the obtained structures are presented in Section 3 and discussed in Section 4. Section 5 contains our concluding remarks.

\section{GENERAL CHARACTERISTICS OF THE PERFORMED SIMULATIONS AND METHODS OF STRUCTURAL ANALYSIS}

The classical Molecular Dynamics method [15-17] consists in numerical solution of equations of motion of many interacting particles. In our simulations the particles were assumed to interact according the Born-Mayer-Huggins potential:

$$
U\left(r_{i j}\right)=A_{i j} \exp \left(-b_{i j} r_{i j}\right)+\frac{Z_{i} Z_{j} e^{2}}{r_{i j}},
$$

with parameterisation (Table 1 in Appendix A) based on [18, 19], using the Lorenz-Berthelot mixing rule (see e.g. [16]). Full ionic charges and periodic boundary conditions along the $x y z$ directions have been used in the simulation.

The glasses were initially prepared as hot melt (at the temperature of $10^{4} \mathrm{~K}$ ), and then slowly cooled to the room temperature (at the average rate of $1.810^{13} \mathrm{~K} / \mathrm{s}$ ), passing intermediate equilibria at the temperatures of $8000,6000,5000,4000,3000,2500,2000,1500$ and $600 \mathrm{~K}$. Velocities were scaled to nominal temperature, $T_{\text {nom }}$, only if the rolling average of temperature, calculated from the moment of the previous scaling, exceeded the $\left(T_{\mathrm{nom}}-\Delta T\right.$, $T_{\text {nom }}+\Delta T$ ) interval, with $\Delta T=100 \mathrm{~K}$ for high-temperature simulations and $\Delta T=10 \mathrm{~K}$ for low-temperature ones. Usually, the last temperature scaling occurred before step 20000 of thermalisation runs. MD runs, specified in Table 2 (Appendix A), were performed using the mdsim code [20].

The direct results of the simulation are the positions and velocities of all the particles at each time-step. The obtained instantaneous or averaged atomic configurations may then be subjected to structural analysis. To describe quantitatively the obtained pPDFs (partial Pair Distribution Functions), the $\Gamma$-like profile has been used [21-23]: 


$$
f(r)=\frac{2 N}{\sigma|\beta| \Gamma\left(4 \beta^{-2}\right)}\left[4 \beta^{-2}+\frac{2(r-R)}{\beta \sigma}\right]^{\left(4 \beta^{-2}-1\right)} \exp \left[-\left(4 \beta^{-2}+\frac{2(r-R)}{\beta \sigma}\right)\right]
$$

where $\mathrm{N}$ is the coordination number, $R$ - average distance, $\sigma$ - standard deviation, and $\beta$ - the skewness parameter.

More detailed analysis of the final atomic configurations, described below, was performed using a number of tool programms contained in the anelli package [24-26].

In order to characterize quantitatively the disorder degree of tetrahedral $\mathrm{GeO}_{4}$ structural units, we use the following shape estimators:

$$
\begin{gathered}
T_{1}=\sum_{i=1}^{6} \frac{\left(\bar{l}_{A-A}-l_{A-A, i}\right)^{2}}{6 \bar{l}_{A-A}^{2}}, \\
T_{2}=\sum_{i=1}^{6} \frac{\left(\bar{l}_{A-A}-l_{A-A, i}\right)^{2}}{6 \bar{l}_{A-A}^{2}}+\sum_{i=1}^{4} \frac{\left(\bar{l}_{C-A}-l_{C-A, i}\right)^{2}}{4 \bar{l}_{C-A}^{2}},
\end{gathered}
$$

where $C$ and $A$ stand for cations and anions, respectively, $\bar{l}_{A-A}$ and $\bar{l}_{C-A}$ mean the average $A-A$ and $C$ - $A$ distances, respectively, and $l_{A-A, i}, l_{C-A, i}$ denote the actual $A-A$ and $C-A$ distances for $i$-th edge of a polyhedron. For ideal polyhedra values of the $T_{1}$ and $T_{2}$ parameters equal zero, whereas for distorted structures they assume positive values.

The distribution of the $T$ values can be calculated for the final MD-simulated structures. In order to interpret such distributions, we compare them with reference data, obtained in the following way (for details see [27]). Assuming the cation's position to be fixed, we shift at random the tetrahedron vertices within spheres (centred on the ideal anion positions) of radius equal to a certain fraction, $r$, of the tetrahedron edge, $L$. For tetrahedra distorted in this way one can calculate the corresponding $T$ values. By repeating the distortion and $T$-calculation cycle many times (say $5 \cdot 10^{6}$ times) for many values of $r$, one gets a $r$-dependent family of $T$ distributions. The reference data so obtained for shape estimators (3) and (4) are shown in Fig. 1. Now, using the minimum square difference criterion, one can individuate a $r$ value that produces the $T$ distribution in best conformity with the MD-simulated $T$ distribution. This means that the distortion degree of the $\mathrm{GeO}_{4}$ tetrahedra can be characterized quantitatively with a single parameter - the best fit value of $r$.

In order to describe the medium-range structure (mutual arrangement of $C A_{n}$ structural units, $C$ - cation, $A$ - anion) one can refer to the second-neighbour partial PDFs ( $C-C$ spatial correlations, i.e. $\mathrm{Bi}-\mathrm{Bi}, \mathrm{Bi}-\mathrm{Ge}$ and $\mathrm{Ge}-\mathrm{Ge})$. However, the interpretation of these correlations is equvocal, and the cation-anion ring statistics seem to characterize the medium-range structure 

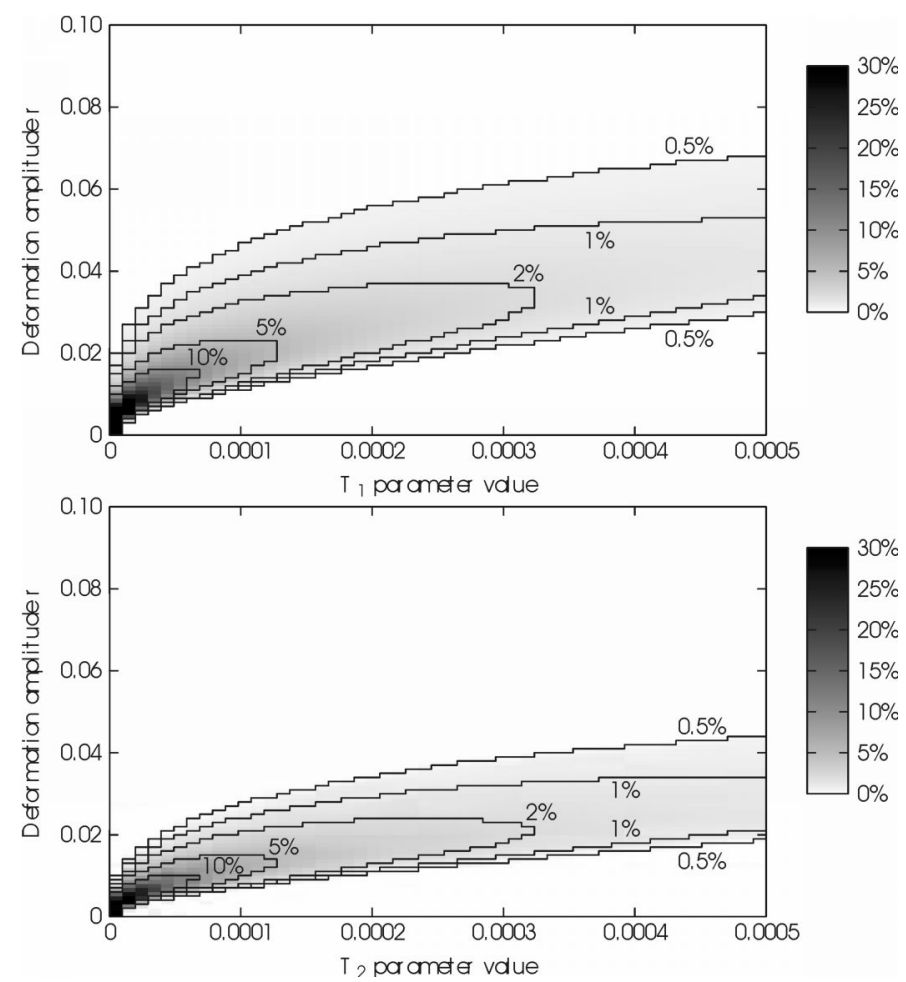

Fig. 1. Distributions of the $T_{1}$ (upper) and $T_{2}$ (lower) estimator values in the function of the deformation amplitude $r$

better. The basal cation-anion rings have been calculated using the Balducci-Perlman-Mancini algorithm [24-26, 28-30].

\section{SIMULATION RESULTS}

Let us now present the structural results referring to the bismuth oxide subsystem (Section 3.1), germania subsystem (Section 3.2) and neutral bismuth atoms (Section 3.3).

\subsection{Bismuth oxide subsystem}

Figure 2 shows the first peak of the spatial $\mathrm{Bi}^{+3}-\mathrm{O}^{-2}$ correlation in the function of $x$ and $p$, together with the $\Gamma$-like components (see Equation (2)). The best fit structural parameters are listed in Table 3 (Appendix B). It is quite clear from Fig. 2 and Table 3 that the first $\mathrm{Bi}^{+3}-\mathrm{O}^{-2}$ PDF peaks are composed of at least two distinct coordination shells, similarly as in pure $\alpha-\mathrm{Bi}_{2} \mathrm{O}_{3}$ [31]. With increasing reduction degree the coordination number related to the first shell, $N_{1}$, increases, whereas $N_{2}$, related to the second coordination shell, decreases. The changes of the coordination numbers are accompanied by systematic variations of the disorder parameters, $\sigma^{2}$ and $\beta$. 


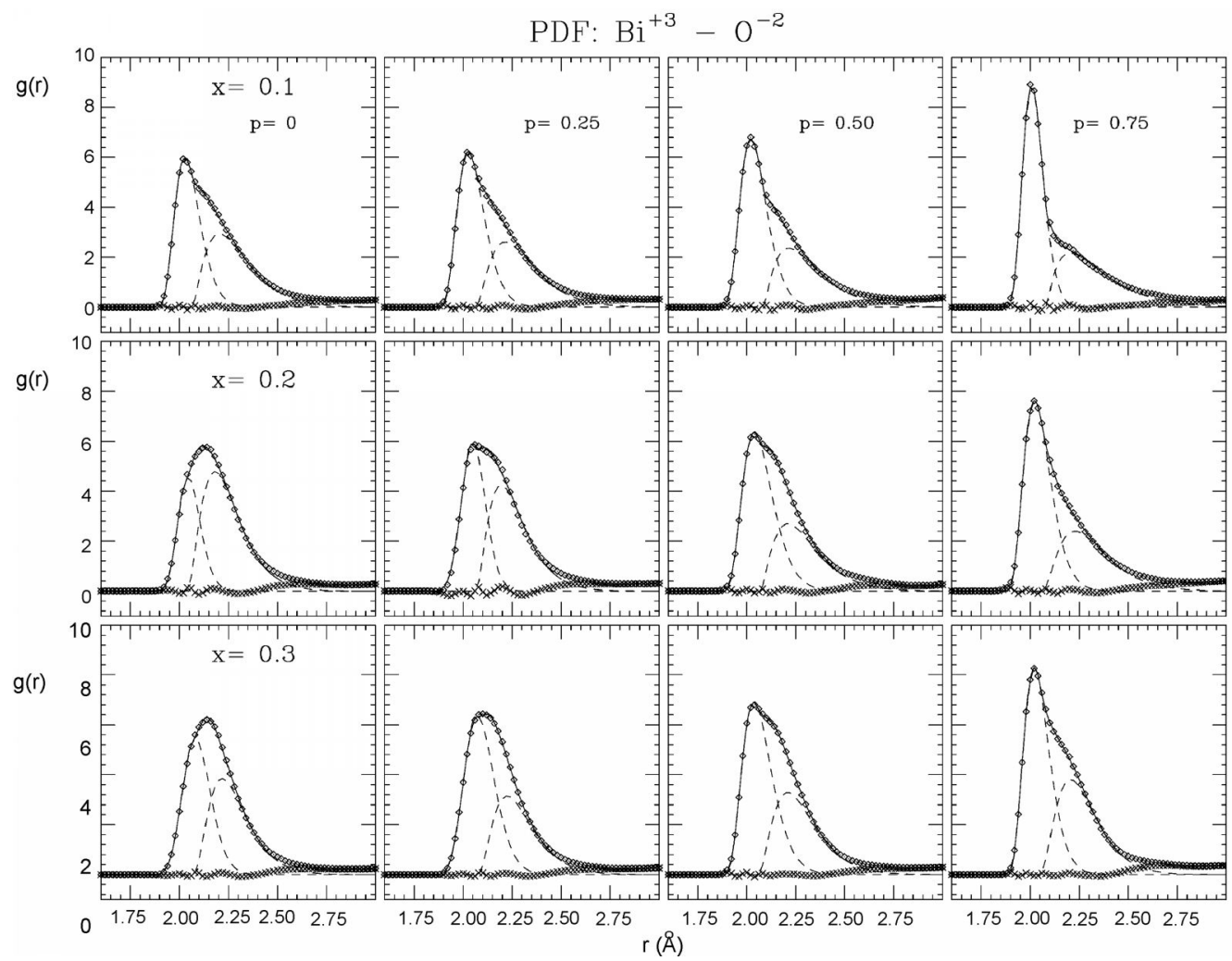

Fig. 2. The first peaks of the spatial $\mathrm{Bi}^{+3}-\mathrm{O}^{-2}$ correlations for $x=0.1,0.2,0.3$, together with the $I$-like components

The spatial arrangement of the oxygen neighbours of bismuth ions can be partially explained analysing the $\mathrm{Bi}^{+3}-\mathrm{O}^{-2}-\mathrm{Bi}^{+3}$ and $\mathrm{O}^{-2}-\mathrm{Bi}^{+3} \mathrm{O}^{-2}$ angular distribution functions calculated for $r_{\text {cut }}=2.6 \AA$. In the $\mathrm{Bi}^{+3}-\mathrm{O}^{-2}-\mathrm{Bi}^{+3}$ angular distribution, a wide peak centred around the angle of $96^{\circ}$ dominates, independently of $\mathrm{x}$ and $p$. A much lower peak centred around $118^{\circ}$ can be also discerned. In the $\mathrm{O}^{-2}-\mathrm{Bi}^{+3}-\mathrm{O}^{-2}$ angular distribution, two wide peaks (of similar intensity) appear at $81^{\circ}$ and $94^{\circ}$ for all $x$ 's and $p$ 's. Significant dispersion of the $\mathrm{Bi}^{+3}-\mathrm{O}^{-2}-\mathrm{Bi}^{+3}$ and $\mathrm{O}^{-2}-\mathrm{Bi}^{+3}-\mathrm{O}^{-2}$ angles suggests a high distortion of the typical $\mathrm{BiO}_{n}$ structural units or a great variety of various local neighbourhoods of the $\mathrm{Bi}$ ion. Thus, the distribution of the $\mathrm{Bi}-\mathrm{O}$ coordination numbers has been calculated. It follows that $\mathrm{BiO}_{5}$ local environments dominate (save for the $x=0.1, p \geq 0.5$ samples), and that with increasing reduction degree, $p$, the contribution of $\mathrm{BiO}_{6}$ units decreases, whereas that of $\mathrm{BiO}_{4}$ units increases. The dominating $\mathrm{BiO}_{5}$ units reveal two morphological types: square pyramids spanned by 5 oxygen ions and triangu- 
lar bipyramids, in both cases with the bismuth ion inside. The square pyramids are twice more frequent than the triangular bipyramids.

In order to get some insight into the medium-range structure of the bismuth subsystem, distributions of $\mathrm{Bi}-\mathrm{O}-\mathrm{Bi}-\mathrm{O}-. .$. rings have been calculated. Remarkable domination of 2-member rings proves that $\mathrm{BiO}_{n}$ units share their edges.

\subsection{Germania subsystem}

The first peaks of $\mathrm{Ge}^{+4}-\mathrm{O}^{-2}$ PDFs are shown in Fig. 3. The one-shell model was used to describe the short-range structure around the germanium cation.
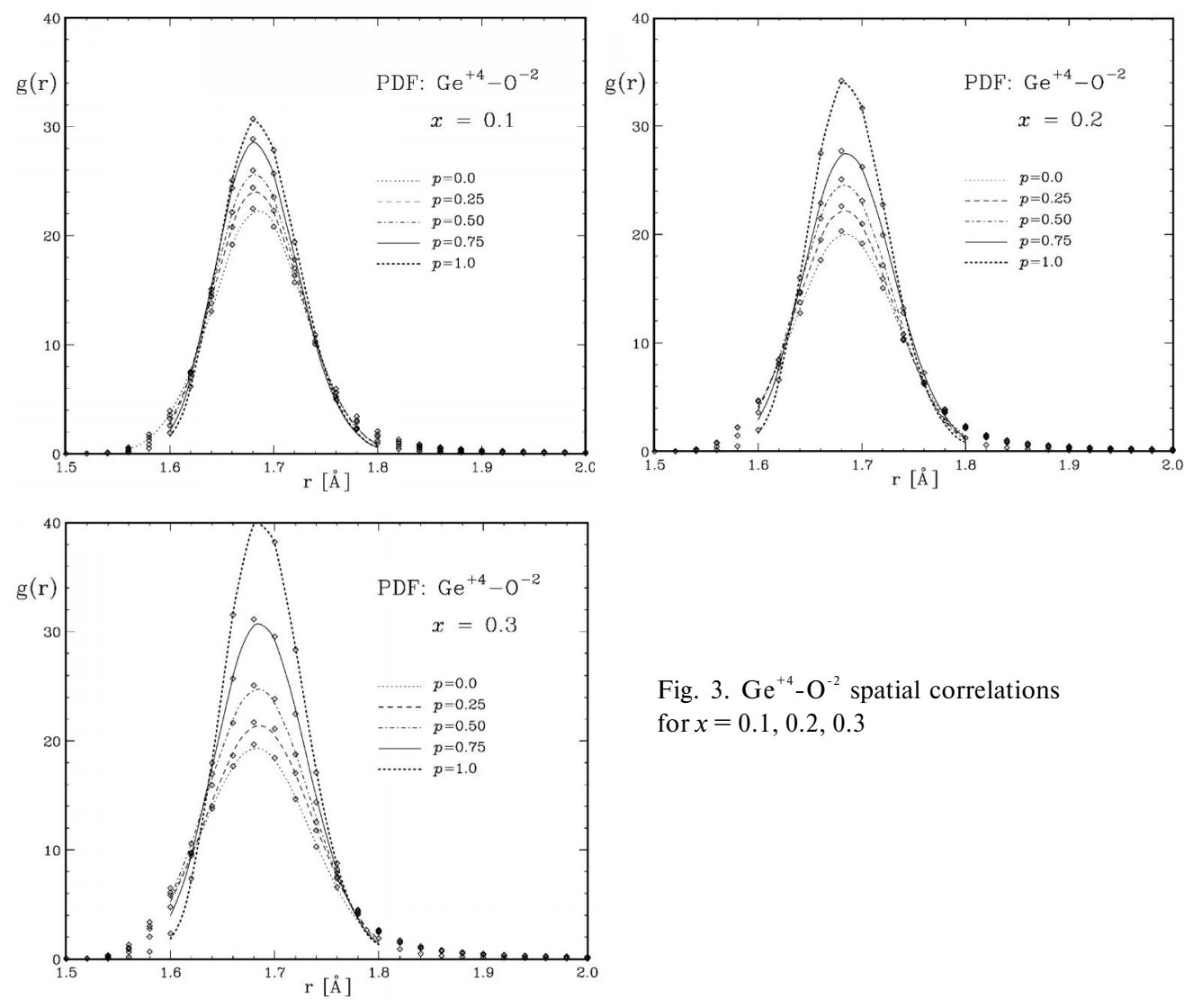

Fig. 3. $\mathrm{Ge}^{+4}-\mathrm{O}^{-2}$ spatial correlations for $x=0.1,0.2,0.3$

Our analysis of the obtained data leads to the following conclusions. The average Ge-O distance does not depend on $x$ or $p$ and amounts to $R=1.69$ A. The average Ge-O coordination number is almost always equal to $4.0 . \mathrm{GeO}_{4}$ units are rather regular (small values of $\sigma^{2}$ and $\beta$ ). However, the peak asymmetry, $\beta$, increases slightly with increasing reduction degree, $p$. 
The determination of the shape of $\mathrm{GeO}_{4}$ units can be completed using the related angular distribution functions. The $\mathrm{O}^{-2}-\mathrm{Ge}^{+4}-\mathrm{O}^{-2}$ angular distribution function shows a single distinct peak at $109^{\circ}$ for all the considered glass compositions and reduction degrees. The $46^{\circ}$ peak, clearly dominating in the $\mathrm{Ge}^{+4}-\mathrm{O}^{-2}-\mathrm{O}^{-2}$ angular distributions, exhibits a similar stability. Such values of the $\mathrm{O}^{-2}-\mathrm{Ge}^{+4}-\mathrm{O}^{-2}$ and $\mathrm{Ge}^{+4}-\mathrm{O}^{-2}-\mathrm{O}^{-2}$ angles are characteristic for regular tetrahedra.

The calculated distributions of the $T_{1}$ and $T_{2}$ parameters fit well the reference data for $r$ equal to about 0.08 and 0.06 , respectively. Thus, the spatial dispersion of oxygen atoms around the ideal positions of tetrahedra vortices can be roughly estimated as $3-4 \%$ of the O-O distance.

In order to recognise the medium-range order, the distributions of $\mathrm{Ge}-\mathrm{O}-\mathrm{Ge}-\mathrm{O}-. .$. ring have been calculated. It turns out that 6-member rings dominate and their contribution increases with increasing $p$, thus the reduction facilitates the relaxation processes in the germania matrix. The basic $\mathrm{GeO}_{4}$ tetrahedral units share their vertices, but do not share their edges (no two-member rings).

\subsection{Neutral bismuth subsystem in modified glasses}

Distributions of neutral $\mathrm{Bi}$ atoms in the last time-step of the simulations are shown in Fig. 4. Apparently, bismuth has aggregated into clusters of various dimensions. The bigger structures are joined together by paths of smaller structures.

Decomposition into three $\Gamma$-like profiles of the first $\mathrm{Bi}^{0}-\mathrm{Bi}^{0}$ peaks has been performed using as the first approximation (initial guess for the optimisation procedure) the data characteristic of crystalline rhomboedric bismuth: $R$ and $N$ equal to $3.104 \AA$ and 3.0, $3.473 \AA$ and 3.0, $4.537 \AA$ and 6.0, respectively. The results are shown in Figure 5. The exact values of the optimal structural parameters are listed in Table 4 (Appendix B). It follows from the data reported therein that the progressing reduction process leads to an increase of the coordination numbers $N_{i}$ (for full reduction, $p=1$, total coordination numbers, $N=N_{1}+N_{2}+N_{3}$, reach values of about $3.6,9.0,10.5$ for $x=0.1,0.2$ and 0.3 , respectively). This suggests an increase of the agglomeration degree. A comparison of the MD-extracted coordination numbers with the values obtained from calculations performed for the spherical and crystalline Bi-granule models has led us to the conclusion that in the $x=0.1$ glass, independently of the modification degree, only $\mathrm{Bi}_{2}$ dimers and $\mathrm{Bi}_{3}$ trimers occur, as in $x=0.2$ after the first modification stage. Increased $x$ and $p$ give rise to increased average granule dimensions up to values above $200 \AA$ (for $x=0.3$ and $p \geq 0.75$ ). The value of $R_{1}$ equal to approximately $3.0 \AA$ in the $x=0.1$ samples confirms the appearance of dimers and trimers (see e.g. [32]). The increase of $R_{1}$ to about $3.1 \AA$ marks the appearance of the bulk bismuth phase. The $R_{2}$ and $R_{3}$ radii of the second and third coordination subshells are in general somewhat shorter than in crystalline Bi. Thus, the structure of confined granules obtained in our simulations is, on average, locally more compact than bulk crystalline bismuth. High values of disorder parameters suggest that our 

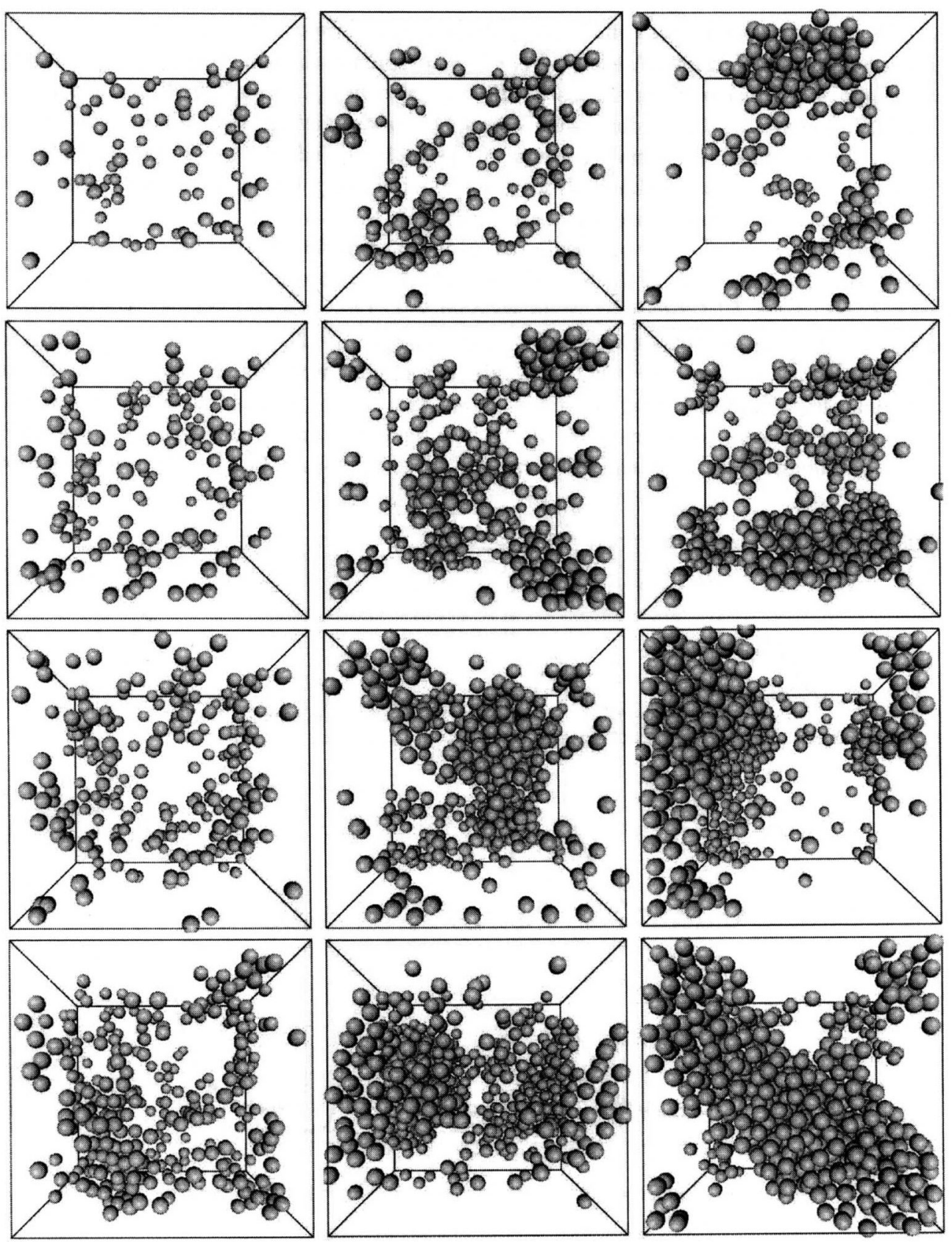

Fig. 4. Spatial distribution of neutral Bi atoms in the last time-step of our simulations (other atoms not shown for clear presentation). The subsequent columns correspond to $x=0.1,0.2,0.3$, respectively, whereas the subsequent rows - to $p=0.25,0.5,0.75,1.0$ 


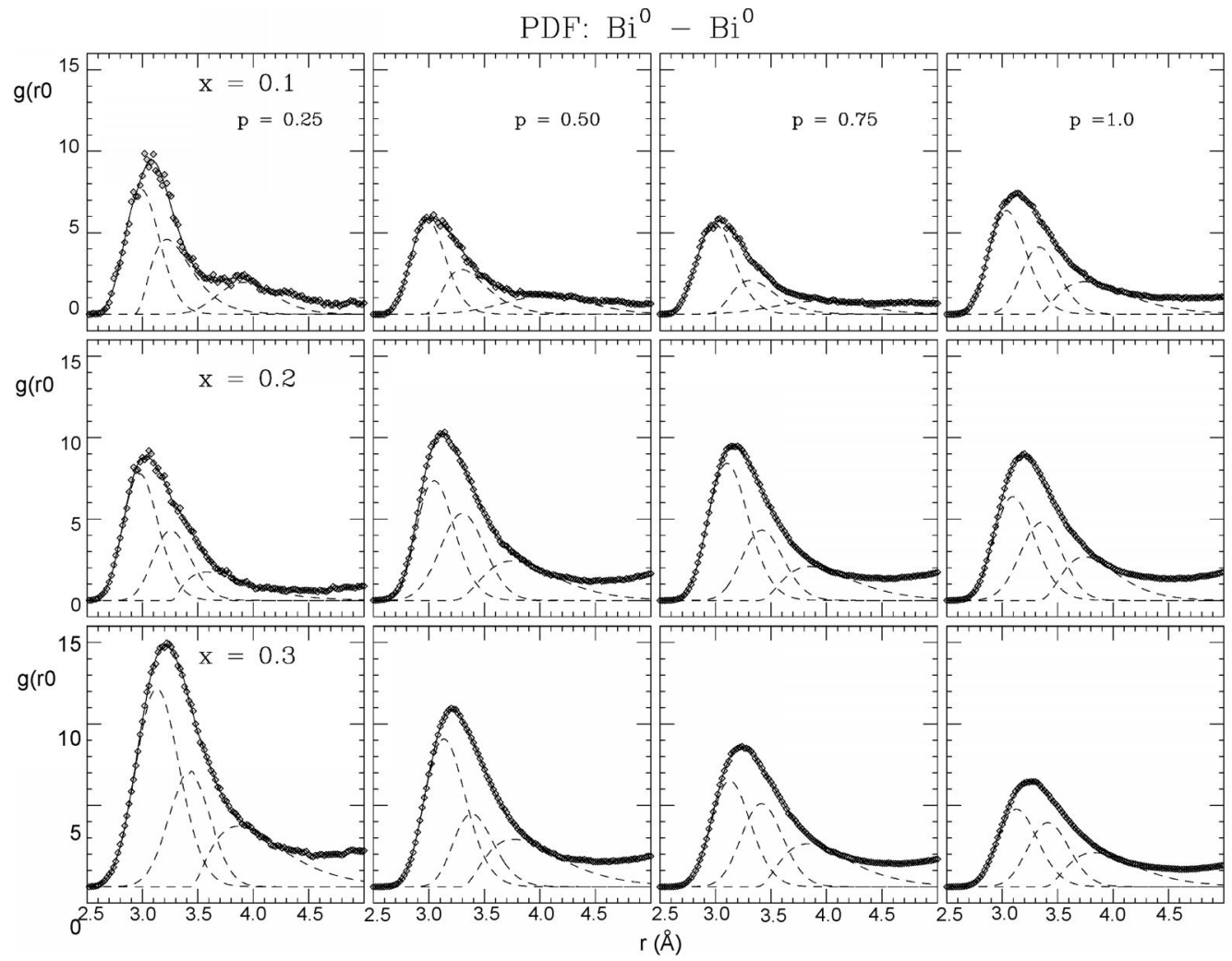

Fig. 5. The first $\mathrm{Bi}^{0}-\mathrm{Bi}^{0}$ peaks together with their $\Gamma$-like components

MD-simulated bismuth granules do not reveal much medium- and long-range order, and may be classified as amorphous structures.

\section{DISCUSSION}

In this section we compare our MD-extracted structural data with the available experimental data on crystalline and glassy BGO compounds (Subsection 4.1) and discuss the similarities and differences in the structure of unmodified and modified BGO glasses and bismuthsilicate (BSO) glasses of the same stoichiometry (Subsection 4.2).

\subsection{Comparison with experimental data}

Let us first confront our MD results with experimental data on $\mathrm{Bi}_{4} \mathrm{Ge}_{3} \mathrm{O}_{12}[5,6], \mathrm{Bi}_{12} \mathrm{GeO}_{20}$ [4] and $\alpha-\mathrm{Bi}_{2} \mathrm{O}_{3}$ [31] crystals.

In all the above-mentioned crystals the local environment of $\mathrm{Bi}$ atoms can be described using a two-shell model, as is the case for our glasses. However, the simulated Bi-O distances 
are usually somewhat shorter than in crystals (the maximum differences are of order of $0.06 \AA$ and $0.3 \AA$ for $R_{1}$ and $R_{2}$, respectively). The O-Bi-O $90^{\circ}$ angles, and some $\sim 66^{\circ}$ angles, detected in the simulated structures, are also observed in crystalline $\mathrm{Bi}_{4} \mathrm{Ge}_{3} \mathrm{O}_{12}$ and $\mathrm{Bi}_{12} \mathrm{GeO}_{20}$. In our simulations the coordination numbers related to the two subsequent $\mathrm{Bi}-\mathrm{O}$ subshells tend with increasing $x$ and $p$ to the values of 3.0 and 2.0 (2.5), respectively, also found in $\mathrm{Bi}_{12} \mathrm{GeO}_{20}$ and $\alpha-\mathrm{Bi}_{2} \mathrm{O}_{3}$ crystalline systems. This indicates the existence of the same $\mathrm{BiO}_{5}$ group as a basic structural unit in glassy and crystalline compounds.

In our glasses, with $\mathrm{x}$ increasing from 0.1 to 0.3 , the $\mathrm{O}$-Bi coordination number increases from 0.4 to 1.3 , whereas the $\mathrm{O}-\mathrm{Ge}$ coordination number decreases from 1.7 to 1.2. Extrapolating these data, one obtains for $x=0.4$ the $\mathrm{O}$ - $\mathrm{Bi}$ coordination equal to 1.8 and the $\mathrm{O}-\mathrm{Ge}$ coordination equal to 1.0. The $\mathrm{O}-\mathrm{Bi}$ and $\mathrm{O}-\mathrm{Ge}$ coordination numbers in $\mathrm{Bi}_{4} \mathrm{Ge}_{3} \mathrm{O}_{12}$ crystal 2.0 and 1.0 , respectively [5].

Tetrahedral GeO4 units, appearing in both considered crystals, are conserved in the glassy state. Thus, the glass' local structure obtained in our simulations resembles the corresponding crystalline phases in many aspects.

Let us compare our results with the data on BGO glasses provided by other authors. According to $[7,8,10]$, the structure of the germania subsystem is $x$-independent and $\mathrm{GeO}_{4}$ tetrahedra are the basic structural units (germanate anomaly is not observed), in full agreement with the present simulation results. However, the simulated Ge-O distance is about $0.03 \AA$ less than in the cited works. According to [8], for higher values of $x$ depolimerisation of $\mathrm{GeO}_{4}$ chains occurs. The same conclusion can be inferred from an analysis of the simulated Ge-O-Ge-O-... ring lengths (a greater value of $x$ has induced a greater number of separated chains and small groups).

As far as the $\mathrm{Bi}_{2} \mathrm{O}_{3}$ subsystem is concerned, $\mathrm{BiO}_{5}$ units dominate in our simulations. This result confirms the results recently obtained from EXAFS measurements [11], and contradicts the data given in [10], where the authors propose deformed $\mathrm{BiO}_{6}$ octahedra as the dominating structural units. The $\mathrm{BiO}_{5}$ groups found in the present simulations and in the EXAFS experiment [11] are characterised by similar values of disorder parameters. The simulated $\mathrm{Bi}-\mathrm{O}$ bond lengths remain somewhat shorter than those inferred from EXAFS [11] (the radii of the first two coordination subshells differ by $0.07 \AA$ and $0.24 \AA$ ).

As far as we are aware, our numerical results concerning the structure of the neutral bismuth subsystem can only be compared with two recent experimental works [11, 14]. These experiments have shown a tendency of neutral $\mathrm{Bi}$ atoms to agglomeration into metallic clusters, just as in our simulations have. The obtained $R$, disorder parameters, $\sigma^{2}$, and coordination numbers, $N$, of neutral Bi atoms' neighbourhood are very similar.

\subsection{Comparison of the structures of bismuth-germanate (BGO) and bismuth-silicate (BSO) glasses}

First of all, it should be noted that the glass-formation ranges are quite different for these compounds: $x \in[0.0,0.3]$ for BGO and $x \in[0.3,0.6]$ for BSO. Thus, despite the $x=0.3$ com- 
position, the comparison will be qualitative in character. The structure of BSO glasses has been recently described in [33, 35].

As far as the structure of unmodified glasses in concerned, one can note that the average $\mathrm{Bi}-\mathrm{O}$ distances are independent of the glass' composition. In BSO glasses they amount to $R_{1} \approx 2.23 \pm 0.01 \AA$ and $R_{2} \approx 2.67 \pm 0.1 \AA$ for the two first coordination subshells. In the simulated BGO glasses $R_{1} \approx 2.08 \pm 0.02 \AA$ and $R_{2} \approx 2.29 \pm 0.03 \AA$. The Bi-O coordination number is only weakly dependent on $x$. In BSO glasses the first subshell contains 3 oxygen atoms, whereas the average occupation of the second subshell increases with $x$ from 1.5 to 2.5 . In the simulated unmodified BGO glasses, $N_{1}$ changes from 2.0 to 2.3 and $N_{2}$ from 2.7 to 2.8 . $\mathrm{BiO}_{5}$ units are the most frequent for all the BGO and BSO glass compositions, and resemble the $\mathrm{BiO}_{5}$ units present in crystalline compounds, mainly in $\alpha-\mathrm{Bi}_{2} \mathrm{O}_{3}$ (see also [36]). $\mathrm{BiO}_{5}$ units are more compact in both glassy systems than in crystalline ones.

With reference to modified BSO and BGO glasses one can note that in both compounds the regularity of the basic structural $\mathrm{BiO}_{5}$ units decreases with increasing reduction degree, and the $\mathrm{SiO} 2$ and $\mathrm{GeO} 2$ networks become more relaxed with the progressing modification process (the occurrence frequency of short rings decreases). In both BGO and BSO glasses, independently of the reduction degree, $\mathrm{BiO}_{n}$ units share their edges, whereas $\mathrm{SiO}_{4}$ and $\mathrm{GeO}_{4}$ units share their vertices. A strong tendency of neutral atoms to agglomeration into clusters has been observed in both compounds, and the metallic subsystem seems to form a $3 \mathrm{D}$ infinite network (big clusters interconnected by small ones).

The $x=0.3$ stoichiometry belongs to the glass formation regions of BGO and BSO glasses, and thus some quantitative comparisons can be performed for this composition. In Table 5 (Appendix C) the structural parameters (EXAFS- and MD-extracted) for the $x=0.3$ BSO glass are quoted after $[33,34]$. Comparing the data reported in Table 5 with those listed in Tables 3 and 4 one can note, that the $\mathrm{Bi}-\mathrm{O}$ distances related to the first coordination subshell (and also to the second coordination subshell, not presented here) are somewhat shorter in germanate glasses, i.e. $\mathrm{BiO}_{n}$ units are more compact in $\mathrm{BGO}$ glasses than in $\mathrm{BSO}$ glasses. It should be noted that MD-extracted structural parameters for BGO and BSO glasses always have values lower than those obtained experimentally. Neutral Bi granules are more compact in the modified $0.3 \mathrm{Bi}_{2} \mathrm{O}_{3} 0.7 \mathrm{GeO}_{2}$ glass than in the $0.3 \mathrm{Bi}_{2} \mathrm{O}_{3} \quad 0.7 \mathrm{SiO}_{2}$ glass $\left(R_{1}\right.$ and other subshell's distances are shorter in $\mathrm{BGO}$ by about $0.25 \AA$ ). Moreover, the $\mathrm{Bi}^{0}-\mathrm{Bi}^{0}$ corresponding disorder parameters, $\sigma^{2}$ and $\beta$ assume lower values in BGO than in BSO. These facts could suggest that the same modification degree should be observed in BGO glass after annealing times longer than those for BSO glasses. Exactly this relation has been observed experimentally in [14].

\section{CONCLUDING REMARKS}

The performed MD simulations enabled us to perform a detailed analysis of the structure of unmodified and modified $x\left[p \mathrm{Bi}_{2}(1-p) \mathrm{Bi}_{2} \mathrm{O}_{3}\right](1-x) \mathrm{GeO}_{2}$ glasses, with $x=0.1,0.2,0.3$ and 
$p=0,0.25,0.5,0.75,1.0$, and the tendencies that accompany the gradually progressing reduction process. Our numerical results agree fairly well with the available experimental data. In conclusion, it should be underlined that in simulated BGO glasses:

- the germanate anomaly is not observed,

- the dominating $\mathrm{BiO}_{5}$ units are more compact in glassy than in crystalline systems,

- reduction leads to the formation of a metallic nano-structure, in the form of a $3 \mathrm{D}$ infinite network,

- the metallic phase in BGO and BSO glasses differs, and this difference could explain the higher modification susceptibility of BSO glasses.

\section{Acknowledgements}

The MD simulations were performed at the TASK Supercomputer Centre (Gdansk, Poland). The work has been partially sponsored by KBN, under grants 7 T11F 01321 and 4 T11F 01925

\section{References}

[1] Nassau K., Chadwick D. L., and Miller A. E., J. Non-Cryst. Solids 93, 115 (1987).

[2] Heo J., Shin Y. B., and Jang J. N., Appl. Opt. 34, 4284 (1995).

[3] Anderson L. P., Grusell E., and Berg S., J. Phys. E: Sci. Instr. 12, 1015 (1979).

[4] Abrahams S. C., Jamieson P. B., and Bernstein J. L., J. Chem. Phys. 47, 4034 (1967).

[5] Radaev S. F, Muradyan L. A., Kargin Y. F, Sarin V. A., Kanepit V. N., and Simonov V. I., Sov. Phys. Crystallogr. 35, 204 (1990).

[6] Omote K. and Waseda Y., J. Non-Cryst. Solids 176, 116 (1994).

[7] Lottici P. P., Manzini I., Antonioli G., Gnappi G., and Montenero A., J. Non-Cryst. Solids 159, 173 (1993).

[8] Capelletti R., Colombi E., Antonioli G., Lottici P. P., Manzini I., Gnappi G., Montenero A., and Parent P., J. Non-Cryst. Solids 177, 170 (1994).

[9] Gaskell P. H., J. Non-Cryst. Solids 222, 1 (1997).

[10] Manzini I., Lottici P. P., and Antonioli G., J. Non-Cryst. Solids 224, 23 (1998).

[11] Zadrożna A., The structure of bismuth/germanate glasses viewed by X-ray absorption spectroscopy, MSc Thesis, Gdansk University of Technology 2003.

[12] Trzebiatowski K., Witkowska A., and Murawski L., Mol. Phys. Rep. 27, 115 (2000).

[13] Kusz B. and Trzebiatowski K., J. Non-Cryst. Solids 319, 257 (2003).

[14] Kusz B., Trzebiatowski K., Gazda M., and Murawski L., J. Non-Cryst. Solids 328, 137 (2003).

[15] Hoover W. G., Molecular Dynamics, Lecture Notes in Physics 258, Springer-Verlag, Berlin, Heidelberg, New York 1986.

[16] Allen M. P. and Tildesley D. J., Computer Simulation of Liquids, Oxford 1997.

[17] Rapaport D. C., The Art of Molecular Dynamics Simulation, Cambridge 1995.

[18] Damodaran K. V., Rao B. G., and Rao K. J., Phys. Chem. Glasses 31, 212 (1990).

[19] Nanba T., Miyaji T., Takada J., Osaka A., Miura Y., and Yasui I., J. Non-Cryst. Solids 177, 131 (1994).

[20] http://www.task.gda.pl/nauka/software

[21] Filipponi A. and Di Cicco A., Phys. Rev. B 51, 12322 (1995).

[22] Filipponi A., J. Phys. CM 13, R23 (2001).

[23] Yang D. S., Fazzini D. R., Morrison T. I., Troger L., and Bunker G., J. Non-Cryst. Solids 210, 275 (1997).

[24] Bergmanski G., Rybicki J., and Mancini G., TASK Quart., 4, 555 (2000).

[25] Rybicki J., Bergmanski G., and Mancini G., J. Non-Cryst. Solids, 293-295, 758 (2001). 
[26] http://www.task.gda.pl/software/anelli

[27] Bergmański G., Białoskórski M., Rybicki J., and Feliziani S., phys. status sol. b (submitted 2004).

[28] Mancini G., TASK Quart., 1, 89 (1997).

[29] Balducci R. and Pearlman R. S., J. Chem. Inf. Comput. Sci., 34, 822 (1994).

[30] Mancini G., J. Phys. Commun. 143, 187 (2002).

[31] Malmros G., Acta Chem. Scan. 24, 384 (1970).

[32] Xu L., Bobev S., El-Bahraoui J., and Sevov C. S., J. Am. Chem. Soc. 122, 1838 (2000).

[33] Witkowska A., Structure of the silicate glasses containing $P b$ and $B i$ atoms viewed by $x$-ray absorption spectroscopy and molecular dynamics simulations, (in Polish), PhD Thesis, Gdansk University of Technology, Gdansk 2002.

[34] Witkowska A., Rybicki J., and Di Cicco A., Phys. Chem. Glasses 43C, 124 (2002).

[35] Witkowska A., Rybicki J., and Di Cicco A., Mol. Phys. Rep. 36, 133 (2002).

[36] George H. B., Vira C., Stehle C., Meyer J., Evers S., Hogan D., Feller S., and Affatigato M., Phys. Chem. Glasses 40 (6), 326 (1999). 


\section{APPENDIX A}

Interatomic potential parameters (Table 1) and the numbers of atoms (Table 2) used in the simulations are listed below.

Table 1. The interatomic interaction parameter used in the present simulations

\begin{tabular}{cccc}
\hline$i-j$ & Symbol & $A_{i j}\left[10^{15} \mathrm{~J}\right]$ & $b_{i j}\left[\AA^{-1}\right]$ \\
\hline $1-1$ & $\mathrm{Bi}^{+3}-\mathrm{Bi}^{+3}$ & 0.4572 & 3.448 \\
$1-2$ & $\mathrm{Bi}^{+3}-\mathrm{Ge}^{+4}$ & 1.0529 & 3.391 \\
$1-3$ & $\mathrm{Bi}^{+3}-\mathrm{O}^{-2}$ & 0.5315 & 3.448 \\
$1-4$ & $\mathrm{Bi}^{+3}-\mathrm{Bi}^{0}$ & 0.4572 & 3.448 \\
$2-2$ & $\mathrm{Ge}^{+4}-\mathrm{Ge}^{+4}$ & 2.4250 & 3.333 \\
$2-3$ & $\mathrm{Ge}^{+4}-\mathrm{O}^{-2}$ & 0.2730 & 3.333 \\
$2-4$ & $\mathrm{Ge}^{+4}-\mathrm{Bi}^{0}$ & 1.0529 & 3.391 \\
$3-3$ & $\mathrm{O}^{-2}-\mathrm{O}^{-2}$ & 0.1889 & 3.153 \\
$3-4$ & $\mathrm{O}^{-2}-\mathrm{Bi}^{0}$ & 0.5315 & 3.448 \\
$4-4$ & $\mathrm{Bi}^{0}-\mathrm{Bi}^{0}$ & 0.4572 & 3.448 \\
\hline
\end{tabular}

Table 2. The numbers of atoms used in our simulations, the edge lenghts of the corresponding simulation

\begin{tabular}{|c|c|c|c|c|c|c|c|}
\hline$x$ & $p$ & $\mathrm{Bi}^{0}$ & $\mathrm{Bi}^{+3}$ & $\mathrm{Ge}^{+4}$ & $\mathrm{O}^{-2}$ & $L[\AA]$ & $\rho\left[\mathrm{g} / \mathrm{cm}^{3}\right]$ \\
\hline \multirow[t]{5}{*}{0.1} & 0.0 & 0 & 320 & 1440 & 3360 & 42.857 & 4.75 \\
\hline & 0.25 & 80 & 240 & 1440 & 3240 & 42.735 & 4.75 \\
\hline & 0.5 & 160 & 160 & 1440 & 3120 & 42.612 & 4.75 \\
\hline & 0.75 & 240 & 80 & 1440 & 3000 & 42.488 & 4.75 \\
\hline & 1.0 & 320 & 0 & 1440 & 2880 & 42.364 & 4.75 \\
\hline \multirow[t]{5}{*}{0.2} & 0.0 & 0 & 600 & 1200 & 3300 & 42.350 & 5.8 \\
\hline & 0.25 & 150 & 450 & 1200 & 3075 & 42.158 & 5.8 \\
\hline & 0.5 & 300 & 300 & 1200 & 2850 & 41.963 & 5.8 \\
\hline & 0.75 & 450 & 150 & 1200 & 2625 & 41.767 & 5.8 \\
\hline & 1.0 & 600 & 0 & 1200 & 2400 & 41.570 & 5.8 \\
\hline \multirow[t]{5}{*}{0.3} & 0.0 & 0 & 840 & 1980 & 3220 & 42.392 & 6.5 \\
\hline & 0.25 & 210 & 630 & 1980 & 2905 & 42.152 & 6.5 \\
\hline & 0.5 & 420 & 420 & 1980 & 2590 & 41.909 & 6.5 \\
\hline & 0.75 & 630 & 210 & 1980 & 2275 & 41.663 & 6.5 \\
\hline & 1.0 & 840 & 0 & 1980 & 1960 & 41.414 & 6.5 \\
\hline
\end{tabular}




\section{APPENDIX B}

Structural parameters describing the first pPDF peaks, referred to in the main text are reported in Tables 3 and 4 below.

Table 3. The best fit structural parameters $R, \sigma^{2}, \beta, N$ of a two-oxygen-shell model of the nearest neighbourhood of $\mathrm{Bi}^{+3}$ in bismuth-germanate glasses

\begin{tabular}{cc|cccc|cccc}
\hline$x$ & $p$ & $R_{1}[\AA]$ & $\sigma_{1}^{2}\left[\AA^{2}\right]$ & $\beta_{1}$ & $N_{1}$ & $R_{2}[\AA]$ & $\sigma_{2}^{2}\left[\AA^{2}\right]$ & $\beta_{2}$ & $N_{2}$ \\
\hline 0.1 & 0.0 & 2.04 & 0.003 & 0.4 & 1.96 & 2.30 & 0.023 & 1.3 & 2.70 \\
& 0.25 & 2.05 & 0.006 & 0.7 & 2.30 & 2.33 & 0.025 & 1.2 & 2.31 \\
& 0.5 & 2.05 & 0.005 & 0.7 & 2.45 & 2.35 & 0.033 & 1.4 & 2.10 \\
& 0.75 & 2.03 & 0.003 & 0.5 & 2.64 & 2.38 & 0.044 & 1.5 & 2.02 \\
\hline \multirow{2}{*}{0.2} & 0.0 & 2.08 & 0.005 & 0.3 & 2.04 & 2.28 & 0.014 & 1.1 & 2.80 \\
& 0.25 & 2.08 & 0.006 & 0.6 & 2.43 & 2.29 & 0.015 & 1.1 & 2.54 \\
& 0.5 & 2.08 & 0.008 & 0.8 & 2.93 & 2.32 & 0.024 & 1.2 & 2.31 \\
& 0.75 & 2.06 & 0.006 & 0.8 & 3.04 & 2.34 & 0.027 & 1.2 & 2.27 \\
\hline \multirow{2}{*}{0.3} & 0.0 & 2.10 & 0.005 & 0.4 & 2.33 & 2.29 & 0.015 & 1.1 & 2.75 \\
& 0.25 & 2.09 & 0.006 & 0.6 & 2.70 & 2.29 & 0.016 & 1.0 & 2.61 \\
& 0.5 & 2.08 & 0.007 & 0.8 & 3.12 & 2.30 & 0.020 & 1.1 & 2.60 \\
& 0.75 & 2.06 & 0.006 & 0.8 & 3.40 & 2.30 & 0.017 & 1.1 & 2.55 \\
\hline
\end{tabular}

Table 4. The best fit structural parameters $\mathrm{R}, \sigma^{2}, \beta, N$ of a three-shell model of the $\mathrm{Bi}^{0}-\mathrm{Bi}^{0}$ spatial correlation in modified bismuth-germanate glasses

\begin{tabular}{ll|cccc|cccc|cccc}
\hline$x$ & $p$ & $R_{1}[\AA]$ & $\sigma_{1}^{2}\left[\AA^{2}\right]$ & $\beta_{1}$ & $N_{1}$ & $R_{2}[\AA]$ & $\sigma_{2}^{2}\left[\AA^{2}\right]$ & $\beta_{2}$ & $N_{2}$ & $R_{3}[\AA]$ & $\sigma_{3}^{2}\left[\AA^{2}\right]$ & $\beta_{3}$ & $N_{3}$ \\
\hline 0.1 & 0.25 & 3.03 & 0.025 & 0.4 & 0.41 & 3.36 & 0.052 & 1.0 & 0.31 & 4.00 & 0.10 & 0.2 & 0.31 \\
& 0.5 & 3.03 & 0.025 & 0.3 & 0.53 & 3.42 & 0.052 & 1.0 & 0.40 & 4.03 & 0.13 & 0.3 & 0.47 \\
& 0.75 & 3.04 & 0.025 & 0.4 & 0.84 & 3.43 & 0.050 & 0.9 & 0.47 & 4.03 & 0.16 & 0.3 & 0.49 \\
& 1.0 & 3.06 & 0.025 & 0.2 & 1.32 & 3.41 & 0.030 & 0.7 & 1.07 & 4.00 & 0.16 & 1.0 & 1.24 \\
\hline 0.2 & 0.25 & .05 & 0.033 & 0.4 & 0.87 & 3.39 & 0.036 & 0.6 & 0.49 & 4.00 & 0.16 & 1.1 & 0.38 \\
& 0.5 & 3.10 & 0.030 & 0.4 & 1.51 & 3.40 & 0.040 & 0.4 & 1.50 & 4.00 & 0.16 & 1.0 & 1.59 \\
& 0.75 & 3.10 & 0.025 & 0.1 & 2.38 & 3.45 & 0.035 & 0.5 & 2.16 & 4.02 & 0.15 & 0.8 & 2.30 \\
& 1.0 & 3.11 & 0.025 & 0.1 & 2.81 & 3.43 & 0.031 & 0.5 & 2.90 & 4.05 & 0.16 & 1.1 & 3.28 \\
\hline 0.3 & 0.25 & 3.13 & 0.032 & 0.2 & 1.67 & 3.41 & 0.034 & 0.3 & 1.68 & 4.10 & 0.19 & 1.2 & 1.87 \\
& 0.5 & 3.15 & 0.029 & 0.3 & 2.42 & 3.42 & 0.035 & 0.5 & 2.20 & 4.08 & 0.20 & 1.2 & 3.03 \\
& 0.75 & 3.16 & 0.029 & 0.2 & 3.06 & 3.45 & 0.033 & 0.4 & 2.95 & 4.06 & 0.16 & 1.0 & 3.86 \\
& 1.0 & 3.16 & 0.029 & 0.2 & 3.00 & 3.44 & 0.035 & 0.4 & 2.96 & 4.05 & 0.18 & 1.0 & 4.50 \\
\hline
\end{tabular}




\section{APPENDIX C}

In Table 5 below, the structural parameters of the $\mathrm{Bi}^{+3}-\mathrm{O}^{-2}$ and $\mathrm{Bi}^{0}-\mathrm{Bi}^{0}$ spatial correlations (only first subshells), previously extracted from EXAFS measurements and MD simulations for the $x=0.3$-BSO glass, are cited after [33, 34].

Table 5. The first subshell $\mathrm{Bi}^{+3}-\mathrm{O}^{-2}\left(R_{l}, \quad \sigma^{2}, \quad N_{1}\right)$ and $\mathrm{Bi}^{0}-\mathrm{Bi}^{0}\left(R_{2}, \sigma_{2}^{2}, \beta_{2}, N_{2}\right)$ structural parameters

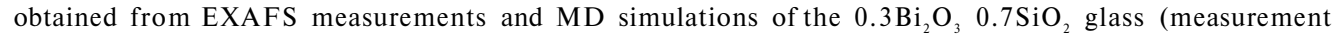
uncertainty is given in parenthesis)

\begin{tabular}{ccc|cccc|cccc}
\hline Method & $x$ & $p / t_{\text {red }}$ & $R_{1}[\AA]$ & $\sigma_{1}^{2}\left[\AA^{2}\right]$ & $\beta_{1}$ & $N_{1}$ & $R_{2}[\AA]$ & $\sigma_{2}^{2}\left[\AA^{2}\right]$ & $\beta_{2}$ & $N_{2}$ \\
\hline \multirow{2}{*}{ MD } & 0.3 & 0.0 & 2.12 & 0.008 & 0.7 & 3.20 & $\mathrm{~B}$ & $\mathrm{~B}$ & $\mathrm{~B}$ & $\mathrm{~B}$ \\
& & 0.25 & 2.11 & 0.010 & 0.9 & 3.35 & 3.24 & 0.030 & 0.1 & 0.91 \\
& 0.5 & 2.10 & 0.010 & 0.9 & 3.15 & 3.33 & 0.040 & 0.2 & 2.24 \\
& 0.75 & 2.03 & 0.004 & 0.6 & 2.34 & 3.34 & 0.046 & 0.3 & 2.75 \\
& 1.0 & - & - & - & - & 3.35 & 0.042 & 0.2 & 3.05 \\
\hline \multirow{2}{*}{ EXAFS } & 0.3 & $0 \mathrm{~h}$ & $2.24(1)$ & $0.034(4)$ & $1.4(3)$ & $3.0(3)$ & - & - & - & - \\
& $1 \mathrm{~h}$ & $2.25(1)$ & $0.059(6)$ & $1.3(3)$ & $1.9(2)$ & $3.38(1)$ & $0.090(9)$ & $1.5(4)$ & $3.8(4)$ \\
& $3 \mathrm{~h}$ & $2.55(1)$ & $0.049(5)$ & $1.6(4)$ & $0.9(1)$ & $3.24(1)$ & $0.059(6)$ & $1.7(5)$ & $3.2(3)$ \\
& $17 \mathrm{~h}$ & $2.56(1)$ & $0.038(4)$ & $1.4(3)$ & $0.8(1)$ & $3.18(1)$ & $0.030(3)$ & $1.4(4)$ & $3.1(3)$ \\
\hline
\end{tabular}

